\title{
Disrupted iron metabolism in peritoneal fluid may induce oxidative stress in the peritoneal cavity of women with endometriosis
}

\author{
Grzegorz Polak', Bartłomiej Barczyński', Iwona Wertel', Wojciech Kwaśniewski', \\ Wiesława Bednarek', Magdalena Derewianka-Polak², Karolina Frąszczak', Marcin Olajossy², \\ Jan Kotarski ${ }^{1}$ \\ ${ }^{1}$ Department of Oncological Gynecology and Gynecology, Medical University of Lublin
${ }^{2}$ Department of Psychiatry and Psychiatry Rehabilitation, Medical University of Lublin
}

Polak G, Barczyński B, Wertel I, Kwaśniewski W, Bednarek W, Derewianka-Polak M, Frąszczak K, Olajossy M, Kotarski J. Disrupted iron metabolism in peritoneal fluid may induce oxidative stress in the peritoneal cavity of women with endometriosis. Ann Agric Environ Med. 2018; 25(4): 587-592. https://doi.org/10.26444/aaem/75802

\begin{abstract}
Introduction and objective. Data on the possible role of peritoneal fluid free radical-mediated oxidative damage in the pathogenesis of endometriosis still remains inconsistent. The aim of the study was to determine iron metabolism markers and their influence on oxidative stress parameters in the peritoneal fluid of women with endometriosis.

Materials and method. 110 women with endometriosis and 119 patients with benign ovarian cysts were included in the study. All visible peritoneal fluid was aspirated during laparoscopy from the anterior and posterior cul-de-sacs. under direct vision to avoid blood contamination. Haemoglobin, iron, total oxidative status, and total antioxidant status were measured using standard colourimetric kits.

Results. Haemoglobin, iron levels, as well as total oxidative status values were significantly higher, whereas total antioxidant status values were significantly lower in the peritoneal fluid of patients with endometriosis, in comparison to the reference groups. No differences were observed in peritoneal fluid concentrations of all parameters measured in relation to the phase of the menstrual cycle.

Conclusions. Peritoneal fluid of women with endometriosis is characterized by disrupted iron metabolism. This is most likely related to an increased number of erythrocytes in the peritoneal cavity of endometriotic women, which leads to a higher concentration of haemoglobin in this environment. Impaired iron homeostasis may have a significant influence on the pathophysiology of peritoneal endometriosis by the direct impact of haemoglobin derivatives and/or formation of the pro-inflammatory and pro-oxidative environment. Peritoneal cavity oxidative stress occurs predominantly in women in advanced stages of the disease.
\end{abstract}

\section{Key words}

Endometriosis, peritoneal fluid, oxidative stress, haemaglobin, iron, total oxidative status, total antioxidant status

\section{INTRODUCTION}

Free radicals have been implicated in the pathogenesis of numerous diseases, including endometriosis. In endometriotic patients pro-oxidant-antioxidant balance may be disturbed by different cells found in the peritoneal cavity (erythrocytes. apoptotic endometrial cells. macrophages), as well as some environmental factors like dioxins and heavy metal ions.

Foyouzi et al. [1] proved that different reactive oxygen species may modulate the growth of endometrial tissue. Pro-oxidant-antioxidant imbalance may contribute to the development of excessive growth of endometrial stromal cells in endometriosis. However, literature data on the possible role of peritoneal fluid (PF) free radical-mediated oxidative damage in the pathogenesis of endometriosis still remain inconsistent.

In 1987, Zeller et al. [2] showed that in women with endometriosis chronic stimulation of the peritoneal cavity resident macrophages by ectopic endometrial implants

Address for correspondence: Grzegorz Polak, Department of Oncological Gynecology and Gynecology, Medical University of Lublin

E-mail: polakg@yahoo.com

Received: 18 June 2016; accepted: 3 April 2017; first published June 2017 provokes constitutive release of large quantities of reactive oxygen products, e.g. superoxide anion, hydrogen peroxide, and singlet oxygen. However, some later studies did not confirm these findings as being clinically significant, showing no differences in the concentration of reactive oxygen forms in both native PF and PF supernatants in endometriotic and healthy women. Free oxygen radicals concentration was not associated with age, menstrual cycle phase, PF volume, or clinical stage [3,4]. Free radicals may show asymmetric distribution in the peritoneal cavity, i.e. increased radicals concentration was detected only locally, close to endometrial implants, but not in the entire peritoneal cavity [5].

It has been proved in animal studies. that the intraperitoneal combined instillation of superoxide dismutase (SOD) and catalase significantly reduced the formation of intraperitoneal adhesions at endometriosis sites [6]. Ota et al. [7] found an increased manganese SOD, copper/zinc SOD, and glutathione peroxidase in the eutopic endometrium of patients with endometriosis, but not in healthy individuals. Furthermore, a significantly lower concentration and activity of SOD was demonstrated in PF of women with endometriosis $[8,9]$, although previous investigations by the authors of the current study [10] and the results of Ishikawa et al. [11] did not confirm this observation. It has also been proved that glutathione 
peroxidase PF activity is lower in women with endometriosis [9]. In a prospective pilot study of infertile women submitted for assisted reproduction procedures, Petean et al. showed a significant decrease in vitamin $\mathrm{E}$ serum levels in a group of patients with endometriosis [12]. Completely opposite results were presented by Jackson et al. [13]. Observations of $\mathrm{PF}$ vitamin E concentration are also inconsistent $[14,15,16]$. Szczepańska et al. [9] demonstrated lower total antioxidant status in PF of infertile women with endometriosis. However, no such difference between endometriotic and healthy individuals was confirmed in a study by Ho et al. [17] and a previous trial by the authors of this study [18].

\section{OBJECTIVES}

The results of trials presented by different authors on the role of oxidative stress phenomenon in endometriosis etiopathogenesis are incoherent, and the conclusions are often contradictory. Thus, the design of the research was to carry out complex investigations on the role of oxidative stress process in endometriosis. The objective of the study was to determine iron metabolism markers and their influence on oxidative stress parameters in PF of women with endometriosis.

\section{MATERIALS AND METHOD}

229 women aged 15-53 who underwent diagnostic or therapeutic laparoscopy were examined. Clinically and histologically confirmed diagnosis established the following groups: women with endometriosis (E. $n=110)$, and as the reference groups, patients with simple serous ( $\mathrm{R} 1, \mathrm{n}=78$ ) and dermoid (R2, n=41) ovarian cysts. In each case. endometriosis was staged according to the American Society for Reproductive Medicine classification [19]. The disease was found to be minimal (E1) in 23 cases, mild (E2) in 25 patients, moderate (E3) in 39 women, and severe (E4) in 23 cases.

Medical history of the patients and basic clinical examination showed no general chronic diseases, except for the condition which was the indication for laparoscopy. Mean age of women did not differ significantly between the studied groups. Similarly, no significant difference was found in the phase of menstrual cycle of the time of laparoscopic procedures between women in all study groups.

All patients signed an informed consent and approval for the study was obtained from the Lublin Medical University Ethics Committee.

All visible PF was aspirated during laparoscopy from the anterior and posterior cul-de-sacs under direct vision to avoid blood contamination. Samples were immediately centrifuged, supernatants were aspirated and stored at -70 $\mathrm{C}$ until analysis. Haemoglobin concentration in the PF was measured in duplicate using a commercially available enzyme-linked immunoassay kit (Imundiagnostik AG. Cat. No. K7816). PF iron levels were determined using the colorimetric kit (Stanbio Iron and Total Binding Capacity (TIBC) - Stanbio Laboratory. Cat. No. 370). Estimation of Total Oxidative Status (TOS) and Total Antioxidant Status (TAS) was performed using commercially available colorimetric kits (PerOx-Immundiagnostic AG. Cat. No. KC5100; ImAnOx- Immundiagnostic AG. Cat. No. KC5200).
All data were tested with the Shapiro-Wilk test for normality. Statistical significance was determined with the Mann-Whitney $\mathrm{U}$ and $\mathrm{H}$ Kruskal-Wallis tests. $\mathrm{P}$ value less than 0.05 was considered statistically significant. Data are presented as medians (Me), minima (Min), maxima (Max). lower and upper quartiles.

\section{RESULTS}

Haemoglobin PF concentrations. Haemaglobin PF levels in patients with endometriosis were significantly higher, compared to women from both reference groups $(\mathrm{p}<0.01)$. Significantly higher PF haemaglobin concentration was observed in patients with all stages of endometriosis, compared to women from both reference groups. No significant difference in PF haemaglobin concentrations was found between women with different endometriosis stages (Fig. 1; Tab. 1).

Table 1. P values for comparisons of haemaglobin PF concentrations between study groups

\begin{tabular}{lcccccc}
\hline \multirow{2}{*}{$\begin{array}{l}\text { Variable: } \\
\text { Haemaglobin }\end{array}$} & \multicolumn{5}{c}{ P values for multiple comparisons (two-sided comparisons) } \\
\cline { 2 - 7 } & R1 & R2 & E1 & E2 & E3 & E4 \\
\hline R1 & & 1.000 & 0.008 & $<0.001$ & $<0.001$ & $<0.001$ \\
\hline R2 & 1.000 & & 0.021 & $<0.001$ & $<0.001$ & $<0.001$ \\
\hline E1 & 0.008 & 0.021 & & 1.000 & 1.000 & 0.486 \\
\hline E2 & $<0.001$ & $<0.001$ & 1.000 & & 1.000 & 1.000 \\
\hline E3 & $<0.001$ & $<0.001$ & 1.000 & 1.000 & & 1.000 \\
\hline E4 & $<0.001$ & $<0.001$ & 0.486 & 1.000 & 1.000 & \\
\hline
\end{tabular}

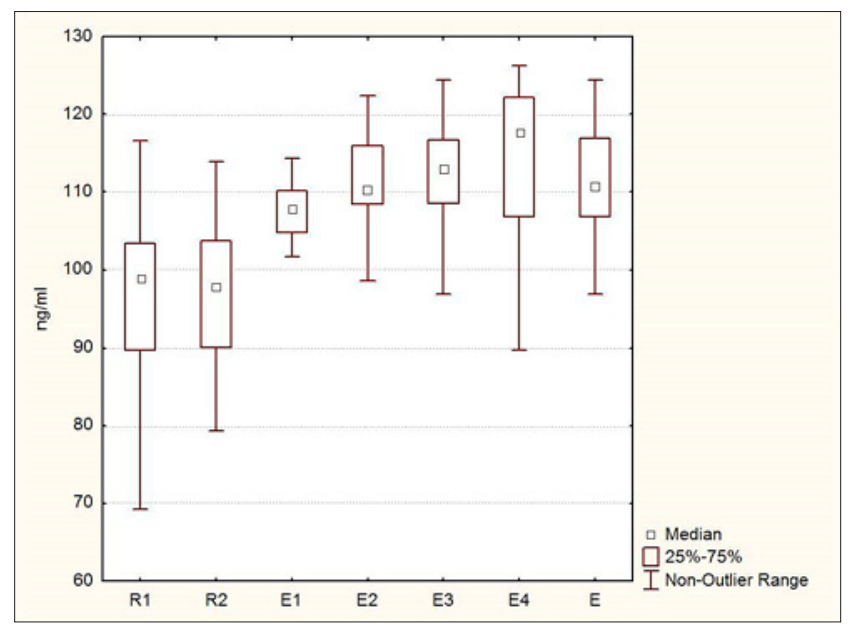

Figure 1. PF concentrations of haemaglobin in the study groups

PF haemaglobin concentration did not differ significantly between the subgroups of women in the follicular and the luteal phase of the menstrual cycle (Me. range; 102, 11.8$126.3 \mathrm{ng} / \mathrm{ml}$ vs. 106.4. 69-241.6 $\mathrm{ng} / \mathrm{ml} ; \mathrm{p}=0.8$ ).

A significant positive correlation $(\mathrm{p}<0.001)$ between PF haemaglobin concentrations and iron levels. and between haemaglobin concentrations and PF TOS. A negative $(\mathrm{p}<0.001)$ correlation was found between PF haemaglobin levels and TAS. 
PF iron levels. Levels of iron were significantly higher $(p<0.01)$ in PF of women with endometriosis, compared to patients with serous and dermoid ovarian cysts. Significantly higher PF concentration of iron was found in women with mild, moderate and severe endometriosis, compared to patients from both reference groups. Patients with minimal endometriosis had higher PF iron levels, compared to women with serous ovarian cysts, but PF iron levels did not differ significantly between women with stage I of endometriosis and subjects with dermoid cysts. Patients with III and IV stages of the disease had significant higher iron levels, compared to women with minimal endometriosis (Fig. 2; Tab. 2).

Table 2. $P$ values for comparisons of iron PF concentrations between study groups

\begin{tabular}{lcccccc}
\hline \multirow{2}{*}{$\begin{array}{l}\text { Variable: } \\
\text { Iron }\end{array}$} & \multicolumn{5}{c}{ P values for multiple comparisons (two-sided comparisons) } \\
\cline { 2 - 7 } & $\mathrm{R} 1$ & $\mathrm{R} 2$ & $\mathrm{E} 1$ & $\mathrm{E} 2$ & $\mathrm{E} 3$ & $\mathrm{E} 4$ \\
\hline R1 & & 1.000 & 0.034 & $<0.001$ & $<0.001$ & $<0.001$ \\
\hline R2 & 1.000 & & 0.516 & $<0.001$ & $<0.001$ & $<0.001$ \\
\hline E1 & 0.034 & 0.516 & & 1.000 & 0.015 & $<0.001$ \\
\hline E2 & $<0.001$ & $<0.001$ & 1.000 & & 1.000 & 0.095 \\
\hline E3 & $<0.001$ & $<0.001$ & 0.015 & 1.000 & & 1.000 \\
\hline E4 & $<0.001$ & $<0.001$ & $<0.001$ & 0.095 & 1.000 & \\
\hline
\end{tabular}

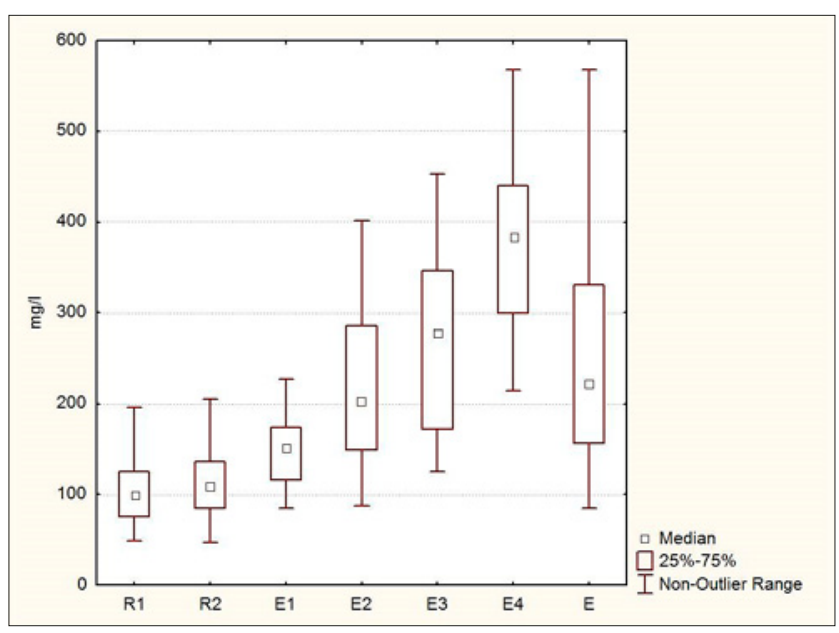

Figure 2. PF concentrations of iron in the study groups

PF iron concentration did not differ significantly between the subgroups of women in the follicular and the luteal phase of the menstrual cycle (Me. range; $138.4,49.1-511.1 \mathrm{mg} / \mathrm{l} \mathrm{vs.}$ 179.8. 46.9-568.4 mg/l; $\mathrm{p}=0.8)$.

A significant positive correlation $(\mathrm{p}<0.001)$ was found between PF iron concentrations and iron levels, and between iron concentrations and PF TOS. Significant negative $(\mathrm{p}<0.001)$ correlation was found between PF haemaglobin levels and TAS.

PF Total Oxidative Status. PF Total Oxidative Status was significantly higher in patients with endometriosis, compared to women with serous and dermoid ovarian cysts. TOS of PF of women with I, III and IV stages of endometriosis were significantly higher, compared to patients from both reference groups $(\mathrm{p}<0.01)$. However, no significant differences
Table 3. $P$ values for comparisons of PF TOS between study groups

\begin{tabular}{lcccccc}
\hline \multirow{2}{*}{$\begin{array}{l}\text { Variable: } \\
\text { TOS }\end{array}$} & \multicolumn{7}{l}{\begin{tabular}{l} 
P values for multiple comparisons (two-sided comparisons) \\
\cline { 2 - 7 }
\end{tabular}} & R1 & R2 & E1 & E2 & E3 & E4 \\
\hline R1 & 1.000 & 1.000 & 0.021 & 0.054 & 0.007 & $<0.001$ \\
\hline R2 & 0.021 & 0.026 & & 1.000 & 1.000 & 1.000 \\
\hline E1 & 0.054 & 0.065 & 1.000 & & 1.000 & 0.386 \\
\hline E2 & 0.007 & 0.015 & 1.000 & 1.000 & & 0.241 \\
\hline E3 & $<0.001$ & $<0.001$ & 1.000 & 0.386 & 0.241 & \\
\hline E4 & & & & & &
\end{tabular}

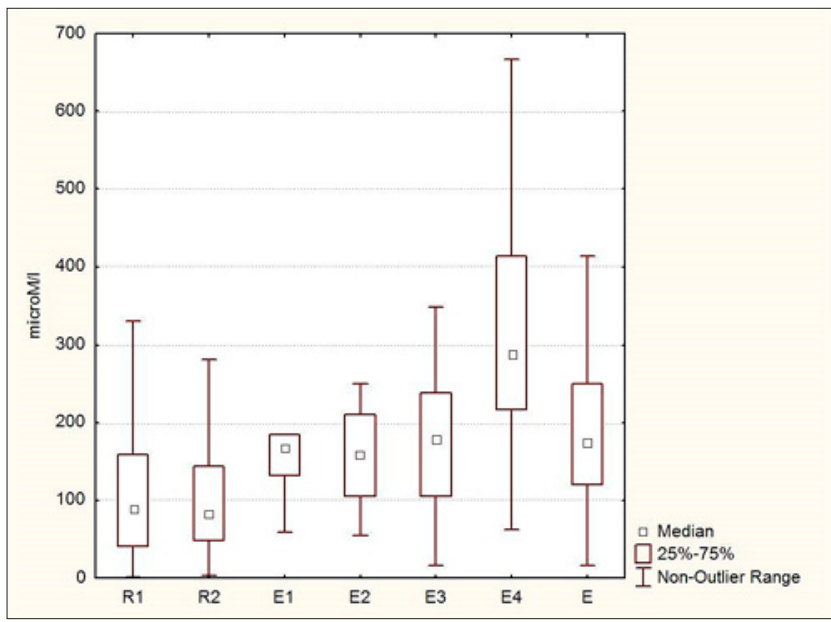

Figure 3. PF TOS in the study groups

were found between PF TAS of women with women with mild endometriosis, and patients form the reference groups (Fig. 3; Tab. 3).

PF TOS did not differ significantly between the subgroups of women in the follicular and the luteal phase of the menstrual cycle (Me. range; 133.5, 3.7-667.4 $\mu \mathrm{mol} / \mathrm{l}$ vs. 120.6, $1.5-625.9 \mu \mathrm{mol} / \mathrm{l} ; \mathrm{p}=0.7)$.

A significant positive correlation $(\mathrm{p}<0.001)$ was found between PF TOS and both iron and haemaglobin levels. Significant negative $(\mathrm{p}<0.001)$ correlation was found between PF TOS and TAS.

PF Total Antioxidant Status. PF Total Antioxidant Status was significantly $(p<0.01)$ lower in women with endometriosis., compared to patients with serous and dermoid ovarian cysts. By analyzing PF TAS in women with different stages of the disease, it was noted that they were lower only in the subgroup of patients with stage IV endometriosis, compared to women from both reference groups. Patients with mild endometriosis had lower PF TAS, compared to women with serous ovarian cysts (Fig. 4; Tab. 4).

PF TAS did not differ significantly between the subgroups of women in the follicular and the luteal phase of the menstrual cycle (Me. range; 391, 41.-1505.3 $\mu \mathrm{mol} / 1$ vs. 370.7, 63-3818.1 $\mu \mathrm{mol} / \mathrm{l} ; \mathrm{p}=0.6)$.

A significant negative correlation $(\mathrm{p}<0.001)$ was found between PF TAS concentrations and TOS, iron, and haemaglobin levels. 
Table 4. P values for comparisons of PF TAS between study groups

\begin{tabular}{lcccccc}
\hline \multirow{2}{*}{$\begin{array}{l}\text { Variable } \\
\text { TAS }\end{array}$} & \multicolumn{7}{c}{ P values for multiple comparisons (two-sided comparisons) } \\
& R1 & R2 & E1 & E2 & E3 & E4 \\
\cline { 2 - 7 } & R1 & 1.000 & 0.089 & 0.893 & 0.004 & $<0.001$ \\
\hline R2 & 1.000 & & 0.344 & 1.000 & 0.051 & 0.007 \\
\hline E1 & 0.089 & 0.344 & & 1.000 & 1.000 & 1.000 \\
\hline E2 & 0.893 & 1.000 & 1.000 & & 1.000 & 0.979 \\
\hline E3 & 0.004 & 0.051 & 1.000 & 1.000 & & 1.000 \\
\hline E4 & $<0.001$ & 0.007 & 1.000 & 0.979 & 1.000 & \\
\hline
\end{tabular}

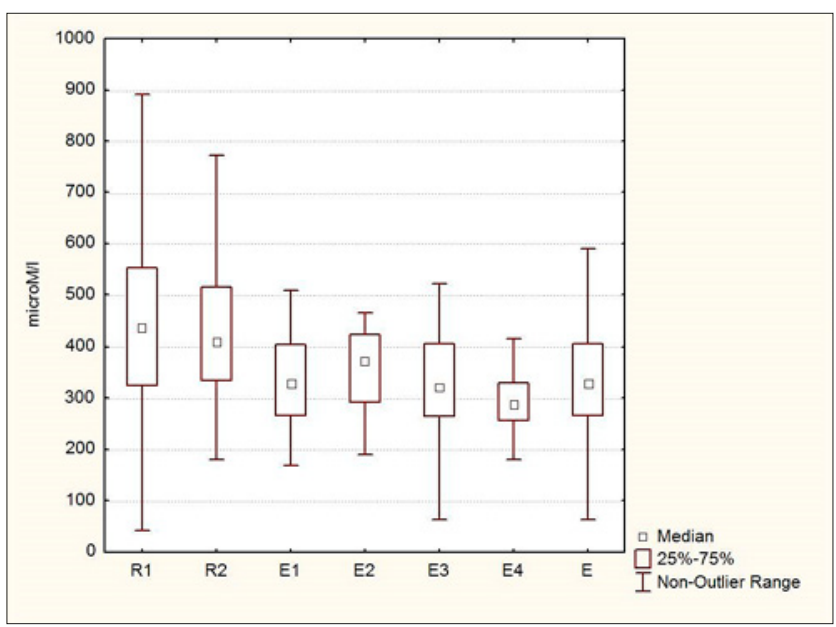

Figure 4. PF TAS in the study groups

\section{DISCUSSION}

According to Sampson's hypothesis [20], the most important process initiating the development of the disease is retrograde menstruation into the peritoneal cavity. Because the phenomenon of 'retrograde menstruation' occurs in most of women at reproductive age, it is claimed that endometriosis results from dysfunctions of mechanisms participating in the elimination of endometrial cells in the peritoneal cavity, erythrocytes, and the products of their decomposition [21].

The presented study demonstrates that PF of women suffering from endometriosis contains a higher concentration of haemaglobin, compared to PF of healthy individuals. The results of this study clearly demonstrate a dysfunction of mechanisms responsible for the elimination of haemaglobin. This observation generally refers to patients with minimal and mild endometriosis in whom bleeding from ectopic implants cannot be intense because of the early stage of the disease. The obtained results suggest that dysfunctions of erythrocytes or haemaglobin elimination from the peritoneal cavity may constitute the fundamental basis of endometriosis pathophysiology.

No significant differences in PF haemaglobin concentration in women in various stages of endometriosis were demonstrated. In patients with minimal and mild disease, with low volume implants, the main source of erythrocytes in the peritoneal cavity is probably the process of 'retrograde menstruation'. However, in advanced stages of the disease, often accompanied by obstruction of oviducts, an essential source of erythrocytes in the peritoneal cavity may be bleeding from endometrial implants. Lack of significant differences in PF haemaglobin concentration between women in different stages of the disease indirectly proves that the concentration of erythrocytes in this environment is similar in all stages of endometriosis.

The degradation of haemaglobin leads to the release of its protein component and heme. Heme's catabolism is related to the formation of many biologically-active substances, including iron ions. In the presented study, a higher concentration of iron was observed in PF of women suffering from endometriosis, compared to the reference groups. These results are in accordance with those obtained by other authors $[5,22,23,24]$. It was also noted that the concentration of iron in PF of women in advanced stages of endometriosis was higher, compared to patients suffering from stage I of the disease. These results are indirectly confirmed by the positive correlation between PF iron concentration. and the stage of endometriosis. demonstrated by Arumugam and Yip [23].

Another important observation is the positive correlation between the concentrations of haemaglobin and iron. This confirms that the main source of iron in the peritoneal cavity is the haemoprotein. Similar to the results obtained by Van Langendonckt et al. [5], significant differences in PF concentrations of both haemaglobin and iron were observed in relation to the phase of the menstrual cycle, which is a very interesting finding. Theoretically, the concentrations of these substances should be increased after menstrual bleeding, i.e. in the follicular phase of the cycle. The results obtained in the presented study are consistent with those of Halme et al. [25] who demonstrated presence of erythrocytes in PF beyond the phase of menstrual bleeding. Similarly, PF haemaglobin and iron concentrations observed in this study indicate the existence of other than 'retrograde menstruation' as the possible source of bleeding within the peritoneal cavity. In women suffering from endometriosis, one of the causes may be endometrial implants. Hypothetically, the presence of these substances in healthy patients in the luteal phase of menstrual cycle may also be explained by ovulation.

Higher PF haemaglobin concentrations in PF of women suffering from endometriosis may have other negative implications. Both free heme and iron ions accumulating in the peritoneal cavity may damage its epithelium, increasing the adhesion of endometrium cells $[5,23]$. Heme, by stimulating the expression of cell adhesion molecules (ICAM1, VCAM-1) and E-selectine in peritoneal and endometrial cells, facilitate the implantation of endometrial cells [26]. Moreover, it was proved that heme may simulate macrophages to produce a wide range of cytokines, including TNF- $\alpha$. IL-1 and IL-6 involved in the pathogenesis of endometriosis [27]. Increased expression of heme oxygenase leads to an increased production of biliverdin, iron ions and carbon monoxide a strong vasodilator, which may stimulate vascularization necessary for the development of implants $[5,28]$. It may also be speculated that a higher concentration of haemaglobin in the PF of women with endometriosis may cause a release of higher amounts of the superoxide radical, and hence induce oxidation stress in the peritoneal cavity.

A very important and pioneer discovery which may constitute a link between iron metabolism and the degree of free radical processes intensity in $\mathrm{PF}$, is a positive correlation between the TOS values and the concentrations of haemaglobin and iron. These results prove a direct relationship between an increased concentration of iron from 
haemaglobin and the intensity of oxidative processes in PF. Results obtained in the presented study clearly demonstrate that the cause of the intensified activity of free radicals in women suffering from endometriosis is an increased concentration of iron. The source of this element is most likely the decomposed erythrocytes entering the peritoneal cavity during 'retrograde menstruation', or released during haemorrhages from the ectopic endometrial implants. In the current study, no differences were found between the TOS of PF, regardless to the phase of the menstrual cycle. Similarly, no differences were found on examining haemaglobin and iron concentrations in PF. In an indirect way, these results confirm the theory that the activity of free radicals in the peritoneal cavity, initiated by haemaglobin and iron, is constant throughout the whole menstrual cycle. This is an interesting observation concerning women with endometriosis. While planning the study, the authors expected that the intensity of these processes, as well as iron PF concentration, would be higher during the follicular phase of the cycle.

The total antioxidant status of the peritoneal fluid of endometriotic patients recruited for this study was significantly lower than in women from the both reference groups. This result proves that the defence potential against free radicals in the peritoneal cavity of such women is significantly lower. This seems to be a natural consequence of increased PF free radical processes. The significant negative correlation between TOS and TAS values demonstrated in the current study confirms the hypothesis. This relationship also demonstrates the strong reliability and validity of the presented data. Increased oxidative processes lead to impairment of the mechanisms of antioxidant defence. The results presented also confirm the results of studies that showed decreased levels of superoxide dismutase, as well as vitamins $\mathrm{C}$ and $\mathrm{E}$, in $\mathrm{PF}$ of women with endometriosis $[12,15,14,29]$, and are consistent with the results obtained by Szczepanska et al. [9] who demonstrated a decrease of the total antioxidant status of PF in endometriotic women. Previous research by the authors of the presented study [18] and a study by Ho et al. [17], showed quite different results. However, they were based on relatively small cohorts of women, therefore, the results obtained in the present study seem to be more representative.

Analyzing the values of PF total antioxidant status of women with endometriosis in relation to the stage of the disease, it was concluded that impairment of the antioxidant processes did not occur in women with minimal and mild disease. It was demonstrated that only PF antioxidant status of women suffering from severe endometriosis was significantly lower, compared to both reference groups. However, in women suffering from stage III of the disease, TAS was significantly lower, compared to patients with serous ovarian cysts. This is an important finding for endometriosis pathophysiology because these results suggest that the mechanisms of antioxidant defence are not impaired by the presence of endometrial implants in early stages of the disease. Thus, it can be speculated that the disturbances of the antioxidant defence occur in advanced disease as a result of a progressive increase in the volume of ectopic endometrial tissue in the peritoneal cavity. The defence defects against free radicals may result from the excessive activity of free radical processes, stimulated by the intensified metabolism of iron in the peritoneal cavity of women with endometriosis.
The impairment of antioxidant mechanisms of PF may not be considered a cause of the disease. but rather a result of its progressive development.

Total antioxidant status of the peritoneal fluid did not differ significantly between the phases of the menstrual cycle. Additionally, as previously described, no differences in PF concentration of free radical processes indicators between the phases of the cycle were found. Taken together, these observations suggest that the parameters of the pro- and anti-oxidant balance of PF are not related to sex hormones changes during the menstrual cycle.

\section{CONCLUSIONS}

PF of women with endometriosis is characterized by disrupted iron metabolism. This is most likely related to an increased number of erythrocytes in the peritoneal cavity of endometriotic women, which leads to a higher concentration of haemaglobin in this environment. This phenomenon may result from a primary defect of the mechanisms responsible for eliminating erythrocytes and their decomposition products, as well as from an increased number of erythrocytes present in the peritoneal cavity that exceeds the capacity of these mechanisms. Impaired iron homeostasis may have a significant influence on the pathophysiology of endometriosis, by direct impact of haemaglobin derivatives and formation of the proinflammatory and prooxidative environment in the peritoneal cavity. It seems that the peritoneal cavity oxidative stress occurs predominantly in women in advanced stages of the disease.

\section{REFERENCES}

1. Foyouzi N, Berkkanoglu M, Arici A, Kwintkiewicz J, Izquierdo D, Duleba AJ. Effects of oxidants and antioxidants on proliferation of endometrial stromal cells. Fertil Steril. 2004; 82: 1019-1022. https:// doi.org/10.1016/j.fertnstert.2004.02.133

2. Zeller JM, Henig I, Radwanska E, Dmowski WP. Enhancement of human monocytes and peritoneal macrophage chemiluminescence activities in women with endometriosis. Am J Reprod Immunol Microbiol. 1987; 3: 78-82. https://doi.org/10.1111/j.1600-0897.1987. $\underline{\mathrm{tb} 00097 . \mathrm{x}}$

3. Wang Y, Sharma RK, Falcone T, Goldberg J, Agarwal A. Importance of reactive oxygen species in the peritoneal fluid of women with endometriosis or idiopathic infertility. Fertil Steril. 1997; 68: 826-830. https://doi.org/10.1016/s0015-0282(97)00343-9

4. Bedaiwy MA, Falcone T, Sharma RK, Goldberg JM, Attaran M, Nelson $\mathrm{DR}$, et al. Prediction of endometriosis with serum and peritoneal fluid markers: a proscpective controlled trial. Hum Reprod. 2002; 17: 426-431. https://doi.org/10.1093/humrep/17.2.426

5. Van Langendonct A, Casanas-Roux F, Donnez J. Iron overload in the peritoneal cavity of women with pelvic endometriosis. Fertil Steril. 2002; 78: 712-718. https://doi.org/10.1016/s0015-0282(02)03346-0

6. Portz DM, Elkins TE, White R, Warren J, Adadevoh S, Randolph J. Oxygen free radicals and pelvic adhesion formation. I Blocking oxygen free radicals toxicity to prevent adhesion formation in an endometriosis model. Int J Fertil. 1991; 36: 39-42.

7. Ota H, Igarashi S, Hatazawa J, Tanaka T. Immunohistochemical assessment of superoxide dismutase expression in the endometrium in endometriosis and adenomyosis. Fertil Steril. 1999; 72: 129-134. https://doi.org/10.1016/s0015-0282(99)00152-1

8. Liu Y, Luo L, Zhao H. Levels of lipid peroxides and superoxide dismutase in peritoneal fluid of patients with endometriosis. J Tongji Med Univ. 2001; 21: 166-167. https://doi.org/10.1007/bf02888087

9. Szczepańska M, Koźlik J, Skrzypczak J, Mikołajczyk M. Oxidative stress may be a piece in the endometriosis puzzle. Fertil Steril. 2003; 79: 1288-1293. https://doi.org/10.1016/s0015-0282(03)00266-8 
10. Polak G, Kozioł-Montewka M, Gogacz M, Kotarski J. Total antioxidant status and activity of an extracellular superoxide dismutase in peritoneal fluid and plasma from women with unexplained infertility. Ginekol Pol. 2000; 71: 571-576.

11. Ishikawa M, Nakata T, Yaginuma Y, Nishiwaki K, Goishi K, Saitoh $\mathrm{S}$. Expression of superoxide dismutase (SOD) in adenomyosis. Am J Obstet Gynecol. 1993; 169: 730-734. https://doi.org/10.1016/00029378(93)90653-Z

12. Campos Petean C, Ferriani RA, dos Reis RM, de Moura MD, Jordao AA Jr, Navarro PA. Lipid peroxidation and vitamin E in serum and follicular fluid of infertile women with peritoneal endometriosis submitted to controlled ovarian hyperstimulation: a pilot study. Fertil Steril. 2008; 90: 2080-2085. https://doi.org/10.1016/j.fertnstert.2007.10.072

13. Jackson D, Craven RA, Hutson RC, Graze I, et al. Proteomic profiling identifies afamin as a potential biomarker for ovarian cancer. Clin Cancer Res. 2007; 13: 7370-7379. https://doi.org/10.1158/1078-0432. ccr-07-0747

14. Murphy AA, Palinski W, Rankin S, Morales AJ, Parthasarathy S. Evidence for oxidatively modified lipid-protein complexes in endometrium and endometriosis. Fertil Steril. 1998; 69: 1092-1094. https://doi.org/10.1016/s0015-0282(98)00087-9

15. Murphy AA, Palinski W, Rankin S, Morales AJ, Parthasarathy S. Macrophage scavenger receptor(s) and oxidatively modified proteins in endometriosis. Fertil Steril. 1998; 69: 1085-1091. https://doi.org/10.1016/ s0015-0282(98)00088-0

16. Seeber BE, Czech T, Buchner H, Bernhart KT, Seger C, Daxenbichler $\mathrm{G}$, et al. The vitamin E-binding protein afamin is altered significantly in the peritoneal fluid of women with endometriosis. Fertil Steril. 2010; 94: 2923-2926. https://doi.org/10.1016/j.fertnstert.2010.05.008

17. Ho HN, Wu MY, Chen SU, Chao KH, Chen CD, Yang YS. Total antioxidant status and nitric oxide do not increase in peritoneal fluids from women with endometriosis. Hum Reprod. 1997; 12: 2810-2815. https://doi.org/10.1093/humrep/12.12.2810

18. Polak G, Kozioł-Montewka M, Gogacz M, Błaszkowska I, Kotarski J. Total antioxidant status of peritoneal fluid in infertile women. Europ J Obstet Gynecol Reprod Biol. 2001; 94: 261-263. https://doi.org/10.1016/ $\underline{\text { s0301-2115(00)00352-3 }}$

19. American Society for Reproductive Medicine. Revised American Society for Reproductive Medicine classification of endometriosis:
1996. Fertil Steril. 1997; 67: 817-821. https://doi.org/10.1016/s00150282(97)81391-x

20. Sampson JA. Peritoneal endometriosis due to the menstrual dissemination of endometrial tissue into the peritoneal cavity. Am J Obstet Gynecol. 1927; 14: 422-469. https://doi.org/10.1016/s00029378(15)30003-x

21. Defrère S, Lousse JC, González-Ramos R, Colette S, Donnez J, Van Langendonckt A. Potential involvement of iron in the pathogenesis of peritoneal endometriosis. Mol Hum Reprod. 2008; 14:377-385. https:// doi.org $/ 10.1093 / \mathrm{molehr} / \mathrm{gan} 033$

22. Arumugam K. Endometriosis and infertility: raised iron concentration in the peritoneal fluid and its effect on acrosome reaction. Hum Reprod. 1994; 9: 1153-1157. https://doi.org/10.1093/oxfordjournals.humrep. a138649

23. Arumugam K, Yip CY. De novo formation of adhesiones in endometriosis: the role of iron and free radical reactions. Fertil Steril. 1995; 64: 62-64.

24. Van Langendonct A, Casanas-Roux F, Eggermont J, Donnez J. Characterisation of iron deposition in endometriotic lesions induced in the nude mouse model. Hum Reprod. 2004; 6: 1265-1271. https:// doi.org/10.1093/humrep/deh182

25. Halme J, Hammond MG, Hulka JF, Raj S, Talbert LM. Retrograde menstruation in healthy women and in patients with endometriosis. Obstet Gynecol. 1984; 64: 151-154.

26. Wagener FAD, Feldman E, De Witte T, Abraham NG. Heme induces the expression of adhesion molecule ICAM-1. VCAM-1 and E selectin in vascular endothelial cells. Proc Soc Exp Biol Med. 1997; 216: 456-463. https://doi.org/10.3181/00379727-216-44197

27. Simoni J, Simoni G, Lox CD, McGunegle DE. Feola M. Cytokines and PAF release from human monocytes and macrophages: effect of haemoglobin and contaminants. ArtifCells Blood Subsit Immobil Biotechnol. 1994; 22: 525-534. https://doi.org/10.3109/10731199409117880

28. Piva M, Sharpe-Timms KL. Peritoneal endometriotic lesions differentially express a haptoglobin-like gene. Mol Hum Reprod. 1999; 5: 71-78. https://doi.org/10.1093/molehr/5.1.71

29. Mier-Cabrera J, Jiménez-Zamudio L, Garcia-Latorre E, HernándezGuerrero C. Quantitative and qualitative peritoneal immune profiles. T-cell apoptosis and oxidative stress-associated characteristics in women with minimal and mild endometriosis. BJOG. 2011; 118: 6-16. https://doi.org/10.1111/j.1471-0528.2010.02777.x 